PPR occurs at the moment of eye opening or closing, and predominantly at a flicker frequency of $15-16 \mathrm{~Hz}$ and wavelength of $700 \mathrm{~nm}$. Inheritance is autosomal dominant. Luminance studies with sunglasses show variable results in the suppression of PPR. A deep red color is required for induction of PPR. In the prevention of the video game Pokemon effect, blue lenses inhibit the PPR by the short wave length and diminished illumination. Seizures evoked by video games are a manifestation of photosensitive epilepsy. During the PPR 75\% patients experience impaired consciousness, opening of eyes or jerking, and pain in the eyes. Persistence of PPR after stimulation is ended is not associated with a higher risk of seizures. Other abnormalities in the EEG, not the PPR are significant in the cause of postPPR seizures. Prognosis is generally good, especially after age 20 years. Valproic acid and levetiracetam are effective in eliminating PPR. (Hughes JR. The photoparoxysmal response: The probable cause of attacks during video games. Clin EEG and Neurosci Jan 2008;39(1):1-7). (Reprints: John R Hughes, MD PhD, University of lllinois Medical Center (M/C 796), 912 S Wood Street, Chicago, IL 60612).

COMMENT. Spikes confined to the occipital region and time-linked with photic stimulation may be a normal finding. Seizures evoked by photic stimulation are usually primary generalized: generalized tonic-clonic, absence, or myoclonic. Photosensitive seizures are classified as pure, occurring only during exposure to photic stimulation, or complicated by spontaneous seizures in addition. Some patients derive a pleasurable response to a self-induced seizure by waving their hands in front of their eyes.

\title{
EYELID MYOCLONIA WITH ABSENCES (JEAVONS SYNDROME)
}

An open-label trial of levetiracetam in 35 patients ( 23 girls) with eyelid myoclonia (EM) was conducted at the Epilepsy Center, Federico II University, Napoli, and several additional epilepsy centers in Italy. Levetiracetam dosage was $10 \mathrm{mg} / \mathrm{kg} /$ day up to $50-60$ $\mathrm{mg} / \mathrm{kg} /$ day in 2 doses in a 12-18 week titration and evaluation phase. Mean dose was 1985 $\mathrm{mg} /$ day. Patients' mean age was $19+/-6$ yrs. Of $28(80 \%)$ patient responders, 6 were seizure-free, 15 had a $>75 \%$ and 7 a $>50 \%$ seizure reduction. Associated generalized tonic clonic seizures (GTCS) in 21 patients were controlled in $14(66 \%)$. The number of days with EM and GTCS was significantly reduced compared to baseline. Paroxysmal abnormalities at eye closure and photoparoxysmal response disappeared or were reduced in 20 responders. Mean follow-up was 24 months. (Striano P, Sofia V, Capovilla G et al. A pilot trial of levetiracetam in eyelid myoclonia with absences (Jeavons syndrome). Epilepsia March 2008;49:425-430). (Respond: Dr Pasquale Striano MD PhD, Epilepsy Center, Frederico II University, Napoli, Italy. E-mail: pstriano@email.it).

COMMENT. Eyelid myoclonia with absences (Jeavons syndrome, 1977) is characterized by the triad of eyelid myoclonia with or without absences (EMA), seizures and EEG paroxysms induced by eye closure, and photosensitivity (Panayiotopoulos 1996, and others), Onset is in childhood, 2-14 years (mean $8 \mathrm{yrs}$ ), especially in girls. Marked intermittent jerking of eyelids after eye closure is associated with jerky upward deviation of eyeballs and retropulsion of the head. Generalized tonic clonic seizures are often associated. AED therapy is usually disappointing, and levetiracetam is the most promising. EMA is not yet recognized as a definite epileptic syndrome by the ILAE. 